\title{
Is there a distinction between malaria treatment and intermittent preventive treatment? Insights from a cross-sectional study of anti-malarial drug use among Ugandan pregnant women
}

Charles O Odongo ${ }^{1,2^{*}}$, Kuteesa R Bisaso ${ }^{1}$, Freddy Kitutu ${ }^{3}$, Celestino Obua ${ }^{4}$ and Josaphat Byamugisha ${ }^{5}$

\begin{abstract}
Background: In Uganda, treatment of clinical malaria and intermittent preventive treatment with sulphadoxinepyrimethamine (SP) are common during pregnancy. As a result, both formal and informal reports from antenatal sources suggest possible misuse of SP for malaria treatment among pregnant women. The objective of this study was to investigate anti-malarial drug use patterns among women who had recently suffered malaria illness before and during pregnancy.
\end{abstract}

Methods: A cross-sectional study in which a structured questionnaire (interviewer-administered) was used to collect data from pregnant women attending an urban antenatal clinic. Details of medicines used to treat malaria episodes suffered before and during pregnancy were captured. A first order Markov probability model was used to estimate probabilities of transitioning between treatment choices made before and during pregnancy. Logistic regression was used to explore whether demographic and obstetric characteristics were associated with transition patterns.

Results: Seven hundred women were interviewed among whom 428 had suffered malaria in both instances. Three hundred thirty of these could recall the medicines used in both instances. Women who used ACT/QNN (correct choice) before pregnancy had higher probabilities of transitioning to SP than staying on ACT/QNN during pregnancy ( 0.463 versus 0.451 ). Access of medicines from private outlets (clinics and pharmacies) were more than nine times predictive of receiving correct medicines ( $p=0.035$ and $p=0.039$ respectively). Access of medicines from clinics was 5.9 times protective against receiving SP for malaria treatment $(p=0.033)$. Among those who used SP before pregnancy, there was a 0.75 probability of staying on it during pregnancy. None of the factors explored could explain this observation.

Conclusion: Use of SP for malaria treatment is common during pregnancy. This may be contributing to adverse pregnancy outcomes. Antenatal care providers should endeavour to emphasize the distinction between treatment and prevention of malaria during pregnancy.

Keywords: Malaria treatment, Intermittent preventive treatment, Sulphadoxine-pyrimethamine, Pregnant women, Uganda

\footnotetext{
* Correspondence: codongo76@gmail.com

'Department of Pharmacology and Therapeutics, School of Biomedical

Sciences, College of Health Sciences, Makerere University, Kampala, Uganda

${ }^{2}$ Department of Pharmacology and Therapeutics, Faculty of Medicine, Gulu

University, Gulu, Uganda

Full list of author information is available at the end of the article
}

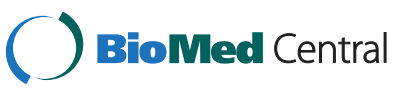

(c) 2015 Odongo et al.; licensee BioMed Central. This is an Open Access article distributed under the terms of the Creative Commons Attribution License (http://creativecommons.org/licenses/by/4.0), which permits unrestricted use, distribution, and reproduction in any medium, provided the original work is properly credited. The Creative Commons Public Domain Dedication waiver (http://creativecommons.org/publicdomain/zero/1.0/) applies to the data made available in this article, unless otherwise stated. 


\section{Background}

Among adult populations in sub-Saharan Africa, pregnant women are disproportionately affected by malaria. The World Health Organization (WHO) recommends a combination of measures to mitigate the adverse impact of malaria during pregnancy. These include; use of insecticide-treated bed nets, early case detection and treatment (with an effective anti-malarial medicine) as well as intermittent preventive treatment during pregnancy (IPTp) [1]. Each year, more than thirty million pregnancies at risk of malaria occur in Africa [2] and of these, Uganda accounts for a considerable proportion mainly for two reasons; First, Ugandan women have the third highest fertility rate in the world [3]. Secondly, malaria is endemic in 95\% of Uganda's geographical territory which include places known to have some of the highest transmission rates ever recorded [4]. While recent efforts to control malaria in Uganda have registered remarkable gains, this dual burden most likely predisposes Ugandan women to high tendencies to consume anti-malarial medicines.

Whereas sulphadoxine-pyrimethamine (SP) remains the most appropriate drug for IPTp, the WHO recommends artemisinin-based combination therapy (ACT) as first-line treatment for uncomplicated malaria in the second and third trimesters of gestation [1]. ACT is however contraindicated in the first trimester of gestation [5] owing to limited safety data on artemisinins in early pregnancy. Instead, oral quinine $(\mathrm{QNN})$ plus clindamycin given for seven days, is the recommended firstline treatment for malaria in this period [1]. The treatment policy for malaria during pregnancy in Uganda is largely similar to that of the WHO [6]. However, effective treatment in the first trimester is often challenging as clindamycin may not be affordable or readily available. This has led to common use of QNN monotherapy with poor adherence due to the relatively long treatment course (7 days) coupled with numerous side effects [7]. Furthermore, contrary to current guidelines, presumptive treatment of malaria remains high in many resourcelimited settings $[8,9]$. This practice has persisted due to continuous challenges with access and delivery of reliable diagnostic services [10]. Needless to say, presumptive treatment leads to wasteful consumption of anti-malarial medicines [9,11] and significantly delays treatment of other febrile illnesses $[11,12]$ and as a result, may portend adverse consequences.

Whereas antenatal care (ANC) clinics have the potential to effectively serve as delivery platforms for malaria control interventions in pregnancy, access to quality ANC remains a challenge in many African settings [13-15]. The Uganda Reproductive Health Policy recommends a minimum of four ANC visits in which a complete package can be delivered [16]. A woman with a normal pregnancy is expected to make at least one visit in each of the first and second trimesters and two visits in the third trimester. During these visits, women are supposed to receive malaria control interventions such as insecticide-treated bed nets, SP- IPTp, and most importantly, knowledge and awareness information on malaria prevention and its treatment. However, both formal $[17,18]$ and informal reports from pregnant women in recent times continue to suggest SP misuse for malaria treatment. The broad objective of this study was to investigate the knowledge and practice gaps associated with malaria treatment among Ugandan pregnant women. This report presents insights from anti-malarial drug use patterns among a population of Ugandan women attending an urban antenatal clinic.

\section{Methods}

\section{Study design}

This was a cross-sectional study that employed quantitative methods of data collection and analysis. Exit interviews were used to collect data from pregnant women attending ANC at Mulago Hospital. Data for this study was obtained as part of a larger cross-sectional study on malaria, IPTp and anti-malarial drug use among Ugandan women, some of which was recently published [19].

\section{Study site and study population}

Mulago Hospital is a 1,500 bed complex located in Kampala, the capital and commercial centre of Uganda. This is a public facility that serves as Uganda's national referral and teaching hospital. In addition to offering a wide range of specialized care, the hospital runs specialized outpatient clinics on week days. Most outpatients come from the urban and peri-urban communities of Kampala and the neighbouring districts of Mukono, Wakiso and Mpigi. The majority of outpatients visiting the hospital tend to come from low and middle income families who prefer to utilize the hospital's free services. The hospital runs two ANC clinics located at Old- and New Mulago complexes. The two clinics offer general (routine) and referral ANC services respectively. The study was conducted at the Old Mulago clinic because it was the first point of call for all pregnant women seeking ANC at the hospital.

\section{Research instrument}

A structured questionnaire developed from our objectives based on the conceptual model proposed by Ribera et al. [20], was used to collect data from participants. Items covered addressing the present objective included a recall of any malaria episode prior to the current pregnancy including the treatment thereof. In addition, if the women had ever suffered from malaria during pregnancy (current or recent pregnancy), the names and sources of 
medicines used were also recorded. If SP was mentioned, care was taken to distinguish between its use for treatment of symptomatic malaria and its use as IPTp (the latter is standard care for all women attending ANC in Uganda [6]). Additional data collected included participant's age, highest education level attained, parity (gestational age $\geq 28$ weeks) and number of ANC visits in their most recent pregnancy. The complete questionnaire is available as Additional file 1. Prior to data collection, the questionnaire was pre-tested on fifty women from the same clinic. This allowed the research team to cater for all possible responses as well as standardize questions so as to ensure internal validity. Detailed data collection methods have been recently published [19].

\section{Sample size determination and selection criteria}

Using the survey formula of Cochran [21], a minimum sample size of 303 participants was required based on the following parameter estimates; a $5 \%$ level of precision, a standard normal deviate $(\mathrm{Z} \alpha)$ of 1.96 (for a confidence level of 95\%) and a $27 \%$ variability level for women who develop malaria during pregnancy based on a recent study conducted in a similar setting [22]. However, in order to qualify for the present analysis, participants had to have experienced malaria before as well as during pregnancy. Therefore, women were consecutively approached without exclusion, and invited to participate in the study. This strategy allowed for random selection and attainment of the desired number of participants.

\section{Recruitment and data collection}

Recruitment and data collection for the entire study was done over three weeks in August 2013. Pregnant women leaving the consultation rooms were consecutively approached and invited to participate. Three female students of nursing were trained and assisted in this exercise. On each clinic day, data collection begun at 09:00 and ended at 13:00 hours. Each research assistant administered the questionnaire to an average of 15 pregnant women per day, with each interview lasting between ten to fifteen minutes. The interviewers were free to ask additional specific questions so as to satisfy themselves that anti-malarial medicines were not being confused with other routine ANC medications. Communication was either in English or a local vernacular language (whichever was convenient for individual women). Whilst in the clinic, all completed questionnaires were scrutinized by the investigators so as to ensure correctness and completeness of records before they were filed.

\section{Ethical considerations}

The study protocol received ethical approval from the hospital's research and ethics committee (Approval Ref.
No. MREC 397) as well as the Uganda National Council for Science and Technology (UNCST). Oral consent was obtained from each participant prior to interviews. Convenience and confidentiality were observed by interviewing each participant in privacy within the clinic premises.

\section{Data management and statistical analysis}

Data was coded, double entered and validated in Epi$D_{a t a}{ }^{\circ}$ software [23] from where it was exported to $\mathrm{R}$ statistical computing software for analysis [24]. In order to analyse drug use patterns for malaria treatment before and during pregnancy, a Markov probability model was proposed. Three main transition states were identified and coded as follows:

State 1-ACT/QNN: Either of these medicines was considered a correct treatment hence the two were combined (since we were unable to determine the actual gestational age at which malaria episode occurred).

State 2-SP: This was considered the misguided choice (arising out of misconception about its indication, possibly due to confusion with its common use as IPTp).

State 3-OTHERS: Consisting of all other incorrect medicines that were mentioned, including chloroquine, antibiotics and herbal remedies.

In order to construct the probability model presented in Figure 1, two assumptions were made namely; i) a constant time interval for each woman, between malaria episodes suffered before and during pregnancy, and ii) each medicine was considered used in its rightful dose and duration at all times. Furthermore, Anderson and Goodman's maximum likelihood ratio criterion [25] was used to test for the Markov property in the data prior to modelling. The null hypothesis was independence of medicine choice before and during pregnancy, while the alternative hypothesis was that choices exhibited partial dependence. With a chi square value of 51 against 4 degrees of freedom, the null hypothesis was rejected $(p<0.000)$. Thus, the data was analysed using a first order Markov model. Transition probabilities were estimated by a simple non-parametric count method. Variables such as 'source of medicine' as well as demographic and obstetric characteristics were investigated as possible predictors of transitioning between states. Multivariate logistic regression analysis of covariate dependence [26] was performed on six transition scenarios which were of interest (see Figure 1) namely;

i. Use of ACT/QNN both before and during pregnancy $(1 \rightarrow 1)$,

ii. Use of ACT/QNN when not pregnant, then transitioning to SP when pregnant $(1 \rightarrow 2)$, 


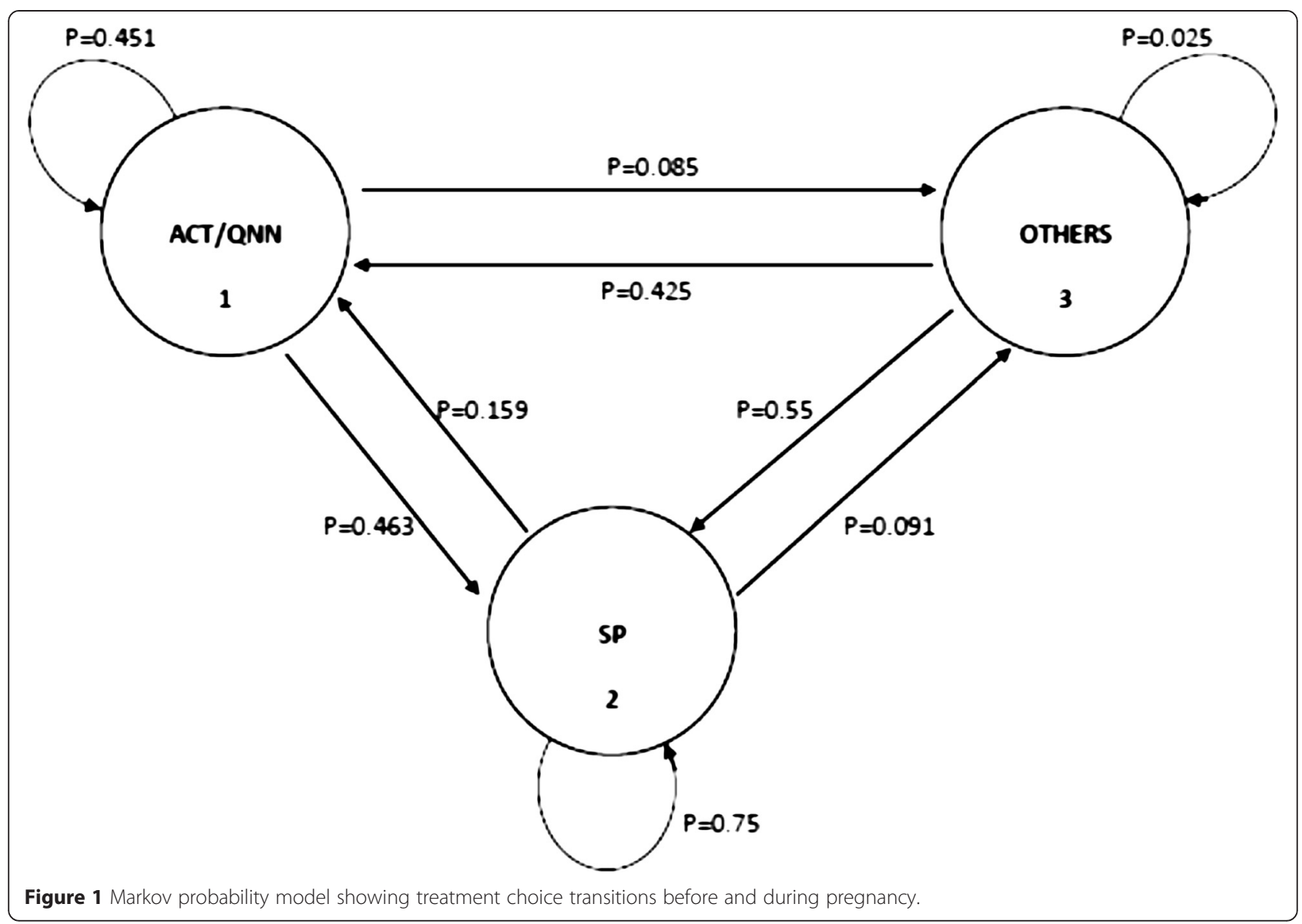

iii. Use of SP both before pregnancy and during pregnancy $(2 \rightarrow 2)$,

iv. Use of SP when not pregnant and transitioning to ACT/QNN when pregnant $(2 \rightarrow 1)$,

v. Use of OTHERS when not pregnant and transitioning to SP when pregnant $(3 \rightarrow 2)$

vi. Use of OTHERS when not pregnant then ACT/QNN when pregnant $(3 \rightarrow 1)$.

Odds ratios were computed for each transition with statistical significance set at $\mathrm{p} \leq 0.05$ at the $95 \%$ confidence level.

\section{Results}

\section{Demographic characteristics of respondents}

Seven hundred respondents were interviewed in order to identify 428 women who had suffered malaria before as well as during pregnancy. Three hundred and thirty of these could correctly recall the medicines used to treat the two malaria episodes and thus constituted the sample for the present analysis. Median age in this group was 25 years (min. 19, max. 41). Most had completed primary $(36.1 \%)$ or basic secondary $(25.2 \%)$ education. Only $24.5 \%$ had attained advanced secondary education or other higher qualifications while $14.2 \%$ had never attended (or failed to complete) primary education. First- and second-time mothers respectively, comprised $16.4 \%$ and $48.4 \%$ of the sample, while women who had experienced higher number of pregnancies comprised 35.1\% of the sample. Excluding first-time mothers, 82.1\% of women had attended between 3-7 ANC visits during their most recent pregnancy. Only $13.6 \%$ had attended 1-2 times while $4.3 \%$ never attended ANC in their most recent pregnancy. When asked to mention the sources of medicines used for treatment of malaria episodes suffered during pregnancy, 50.0\% $(n=165)$ reported accessing medicines from a private clinic while $29.1 \%$ accessed from a hospital. Other sources of medicines were public health centres $8.5 \%$, private pharmacies $8.8 \%$, and community drug shops $3.6 \%$.

State occupancy and transition probabilities for medicines used before and during pregnancy

Overall, ACT/QNN were the most commonly used treatment before pregnancy while SP was most commonly used during pregnancy. This was true regardless of the medicines used before pregnancy. Table 1 presents state prevalence and probability estimates for use 
Table 1 State prevalence and transition probability matrix for anti-malarial treatments used before and during pregnancy

\begin{tabular}{llllll}
\hline & \multicolumn{2}{l}{ State prevalences $(\%) \mathbf{n}=\mathbf{3 3 0}$} & & \multicolumn{2}{l}{ Transition probabilities } \\
\cline { 2 - 3 } \cline { 5 - 6 } & Before pregnancy & During pregnancy & & ACT/QNN & $\rightarrow$ Others \\
\hline ACT/QNN $\rightarrow$ & 74.5 & 41.9 & 0.451 & 0.085 & 0.463 \\
Others $\rightarrow$ & 12.1 & 7.9 & 0.425 & 0.025 & 0.550 \\
SP $\rightarrow$ & 13.3 & 51.2 & 0.159 & 0.091 & 0.750 \\
\hline
\end{tabular}

of anti-malarial medicines before, then during pregnancy. Figure 1 presents the transition probability estimates (from Table 1) in form of a Markov model diagram. Among women who used ACT/QNN before pregnancy, there was a higher probability of switching to SP $(\mathrm{p}=0.463)$ than remaining on $\mathrm{ACT} / \mathrm{QNN}(\mathrm{p}=0.451)$ during pregnancy. Among those who used SP before pregnancy, there was a higher probability of remaining on SP $(\mathrm{p}=0.75)$ than switching to ACT/QNN $(\mathrm{p}=0.159)$ during pregnancy. Among those who used 'OTHERS' before pregnancy, there was a higher probability of switching to SP $(\mathrm{p}=0.55)$ than switching to ACT/QNN ( $\mathrm{p}=0.425)$.

\section{Factors determining treatment choice transitions during pregnancy}

Of the six transition scenarios for which multivariate analysis was performed, significant findings were seen in only two scenarios; 1 : use of $\mathrm{ACT} / \mathrm{QNN}$ in both instances, before and during pregnancy $(1 \rightarrow 1)$, and 2 : use of ACT/QNN before pregnancy then switching to SP during pregnancy $(1 \rightarrow 2)$. Of all predictor variables investigated, only 'source of medicine' significantly predicted transitions in the two scenarios. Women who used ACT/QNN prior to pregnancy were 9.8 and 11.4 times likely to stay on it if the medicine was accessed from a private clinic or private pharmacy respectively (Table 2). Among women who used ACT/QNN before pregnancy, accessing medicines from a private clinic was 5.9 times protective against switching to SP during pregnancy. A similar trend was noticed with all other sources of medicines although they did not reach statistical significance (see Table 2).

\section{Discussion}

This study used a transition probability model to show changes in malaria treatment patterns among pregnant women in Uganda. Women were more likely to use SP than $A C T / Q N N$, to treat reported episodes of malaria during pregnancy. The prevalence and probability patterns revealed here show that women (or their treatment providers) consciously chose to use SP for malaria treatment, highlighting possible existence of confusion about its role (indication) during pregnancy. High levels of awareness about SP as an anti-malarial drug coupled with low knowledge about its indication during pregnancy has been previously reported in this study population [19]. This critical knowledge deficit may be attributed to the low education levels considering that more than half of the women had received limited (primary) or no formal education. It is, therefore, probable that they did not easily comprehend the distinction between treatment and prevention of malaria and the drugs thereof. Because patients in developing countries most often trust and follow malaria treatment advice offered by health workers [27], these results may be a reflection of health workers' lack of focussed malaria treatment information. Alternatively, health workers may be delivering this information in a manner that is inappropriate to recipients' needs, given their low literacy and low knowledge base, thus leaving them 'half-baked'.

Many previous studies have highlighted the high rates of self-medication and informal treatment-seeking behaviour in Uganda [27-30]. In light of these considerations, the present findings raise two disturbing concerns; first, unless SP-IPTp is administered under the strict supervision of a health worker, women who do not perceive themselves as ill with malaria (as at routine ANC visits), are unlikely to take SP-IPTp by themselves. As such, questions as to whether pregnant women comply with unsupervised intake (of SP-IPTp) remain valid [19]. Secondly, SP lacks efficacy against clinical malaria. Its use for malaria treatment may, therefore, be associated with high risks of treatment failure and the consequences thereof. These concerns may not be far-fetched considering the high awareness about SP, its inexpensiveness and wide availability in many drug outlets within Uganda, often with inadequate regulatory oversight. It is, therefore, possible that consequences of inappropriate use of SP significantly contribute to the high incidences of poor pregnancy outcomes in Uganda. In a recent study from Mukono district (Uganda), Mbonye et al. showed a high prevalence of self-medication with SP among cotrimoxazole-taking HIV positive pregnant women presenting with a febrile illness [17]. Clearly, such practices underscore the misconception about SP as a treatment for malaria during pregnancy.

As with many studies from Uganda and elsewhere [29-33], private drug outlets were an important source of anti-malarial medicines in this study. This may be due to their common location within residential areas where 
Table 2 Multivariate regression analysis of predictors of transitioning between malaria treatments received before and during pregnancy

\begin{tabular}{|c|c|c|c|c|c|}
\hline Variables & Log odds & Std. error & $\mathrm{OR}^{*}(\mathrm{C} . \mathrm{I})$. & $1 / \mathrm{OR}^{\dagger}$ & p-value \\
\hline \multicolumn{6}{|c|}{ Transition scenario ACT/QNN before and during pregnancy $(1 \rightarrow 1)$} \\
\hline Constant & -1.298 & 1.461 & 0.273 & & 0.374 \\
\hline Age & -0.032 & 0.043 & $0.969(0.89-1.05)$ & 1.03 & 0.456 \\
\hline Education level & 0.049 & 0.119 & $1.05(0.83-1.34)$ & & 0.678 \\
\hline Parity & -0.107 & 0.135 & $0.899(0.69-1.68)$ & 1.11 & 0.423 \\
\hline No. of ANC visits & 0.011 & 0.098 & $1.011(0.83-1.23)$ & & 0.913 \\
\hline Private clinic & 2.286 & 1.085 & $9.84(1.69-18.6)$ & & 0.035 \\
\hline Pharmacy & 2.429 & 1.178 & $11.35(1.53-23.8)$ & & 0.039 \\
\hline Public Health center & 1.195 & 1.170 & $3.3(0.44-6.8)$ & & 0.307 \\
\hline Hospital & 2.009 & 1.098 & $7.46(1.24-14.3)$ & & 0.067 \\
\hline \multicolumn{6}{|c|}{ Transition scenario ACT/QNN to SP before and during pregnancy $(1 \rightarrow 2)$} \\
\hline Constant & 0.267 & 1.267 & $1.31(0.11-18.13)$ & & 0.833 \\
\hline Age & 0.021 & 0.043 & $1.02(0.94-1.11)$ & & 0.626 \\
\hline Education level & -0.038 & 0.119 & $1.04(0.76-1.22)$ & & 0.754 \\
\hline Parity & 0.169 & 0.134 & $1.18(0.91-1.55)$ & & 0.208 \\
\hline No. of ANC visits & 0.089 & 0.098 & $1.09(0.90-1.33)$ & & 0.359 \\
\hline Private clinic & -1.776 & 0.834 & $0.169(0.02-0.75)$ & 5.9 & 0.033 \\
\hline Pharmacy & -1.684 & 0.954 & $0.186(0.02-1.08)$ & 5.4 & 0.078 \\
\hline Public Health center & -1.504 & 0.915 & $0.222(0.03-1.19)$ & 4.5 & 0.10 \\
\hline Hospital & -1.506 & 0.850 & $0.221(0.03-1.02)$ & 4.4 & 0.076 \\
\hline
\end{tabular}

${ }^{*}$ OR: odds ratio, ${ }^{+}$inverse operation applied to OR estimates less than 1 only.

boldface figures indicate statistical significance at $\mathrm{p}$ less than 0.05 at the $95 \%$ confidence level.

they act as points of first call for remedies to acute illness. Interestingly in this study, access of anti-malarial medicines through private drug outlets appeared to guard against inappropriate use of SP. This finding is contrary to past studies that have suggested that treatment decisions among private or informal health care providers were often irrational, driven more by profit motives than clients' interests [34]. Despite the fact that we did not determine actual prescribers of the medicines reported in this study, the possibility of self-treatment cannot be under-estimated since the purchase of medicines without a prescription is not uncommon in this society as cited above. Evidence from previous studies suggests that both patients and prescribers alike, contribute to the culture of irrational drug-use. In the study from Mukono cited above, $10.9 \%$ of parasite-confirmed and $48.6 \%$ of parasite-negative pregnant women received SP as treatment from health centre staff [17]. Therefore, both parties may be responsible for the irrational pattern of SP use observed in this study.

The findings from this study may have two important limitations, namely respondents' ability to distinguish malaria from other illnesses as well as recall bias to do with event details. Whereas nearly all data collected in this study reflected events that happened more than one month earlier, malaria is not just an event in this society, rather, it is a very common experience that respondents easily relate with. As such, many adults, especially household heads (as in this case) have vivid ideas on its symptoms and popular remedies. Therefore, accurate memory about the details around malaria episodes were likely to be high. Furthermore, only respondents who could clearly identify anti-malarial medicines by name or description were included in this analysis. Ninety-eight potential respondents who failed to clearly articulate names or descriptions of the medicines used in both states were excluded. Thus, the above limitations were significantly minimized within the limits of the cross-sectional study design and the research tools employed.

\section{Conclusion}

This study highlights the inappropriate use of SP for malaria treatment among pregnant women in Uganda. In order to address this problem and improve malaria treatment during pregnancy, health workers should at all times endeavour to provide appropriate information on malaria treatment. Additional emphasis should be placed on the distinction between prevention and treatment of clinical malaria during pregnancy. 


\section{Additional file}

Additional file 1: Knowledge and experiences with malaria and sulphadoxine-pyrimethamine (Fansidar) among pregnant women at Mulago referral hospital, Kampala. This is a three-part, twenty-item, research instrument (questionnaire) used to collect data on knowledge and experiences with malaria and anti-malarial drug use among Ugandan pregnant women.

\section{Abbreviations}

ACT: Artemisinin combination therapy; ANC: Antenatal care

IPTp: Intermittent preventive treatment of malaria during pregnancy; QNN: Quinine; SP: Sulphadoxine-pyrimethamine; WHO: World Health Organization.

\section{Competing interests}

The authors declare that they have no competing interests.

\section{Authors' contributions}

COO participated in conception and design of the study, data collection, analysis and interpretation, and drafting of the manuscript. RBK participated in data analysis, interpretation, read and critically revised the manuscript. FK, $\mathrm{CO}$ and JB participated in conception and design of the study, read and critically revised the manuscript. All authors approved the final version of the manuscript.

\section{Acknowledgements}

This work was supported by Grant Number 5R24TW008886 supported by the following offices of the United States of America; Office of the US Global Aids Coordinator (OGAC), National Institutes of Health $(\mathrm{NIH})$ and Health Resources and Services Administration (HRSA). Its contents are solely the responsibility of the authors and do not necessarily represent the official views of the supporting offices. We are sincerely grateful to Komujuni Sarah Caro, Agasha Anita and Wahab Sofia for their diligent services as interviewers. We thank all the women who kindly accepted to participate in this study.

\section{Author details}

'Department of Pharmacology and Therapeutics, School of Biomedical Sciences, College of Health Sciences, Makerere University, Kampala, Uganda. ${ }^{2}$ Department of Pharmacology and Therapeutics, Faculty of Medicine, Gulu University, Gulu, Uganda. ${ }^{3}$ Department of Pharmacy, School of Health Sciences, College of Health Sciences, Makerere University, Kampala, Uganda. ${ }^{4}$ Mbarara University of Science and Technology, Mbarara, Uganda. ${ }^{5}$ Department of Obstetrics and Gynaecology, School of Medicine, College of Health Sciences, Makerere University, Kampala, Uganda.

Received: 20 October 2014 Accepted: 20 April 2015 Published online: 03 May 2015

\section{References}

1. WHO. World malaria report 2012. Geneva, Switzerland: World Health Organization; 2013.

2. Dellicour S, Tatem A, Guerra C, Snow R, ter Kuile F. Quantifying the number of pregnancies at risk of malaria in 2007: a demographic study. PLoS Med. 2010;7:e1000221.

3. Uganda total fertility rate - demographics. Available from [www.indexmundi. com/uganda/total_fertility_rate.html]. Last accessed October 152014.

4. Okello PE, Van Bortel W, Byaruhanga AM, Correwyn A, Roelants P, Talisuna $A$, et al. Variation in malaria transmission intensity in seven sites throughout Uganda. Am J Trop Med Hyg. 2006;75:219-25.

5. Dellicour S, Hall S, Chandramohan D, Greenwood B. The safety of artemisinins during pregnancy: a pressing question. Malar J. 2007;6:15.

6. National Malaria Control Programme. Uganda National Malaria Contro Policy 2011. Kampala, Uganda: Ministry of Health; 2011.

7. Yeka A, Achan J, D'Alessandro U, Talisuna A. Quinine monotherapy for treating uncomplicated malaria in the era of artemisinin-based combination therapy: an appropriate public health policy? Lancet Infect Dis. 2009;9:448-52
8. Omo-Aghoja LO, Abe E, Feyi-Waboso P, Okonofua FE. The challenges of diagnosis and treatment of malaria during pregnancy in low resource settings. Acta Obstet Gynaecol Scand. 2008;87:693-6.

9. Batwala V, Magnussen P, Hansen KS, Nuwaha F. Cost-effectiveness of malaria microscopy and rapid diagnostic tests versus presumptive diagnosis: implications for malaria control in Uganda. Malar J. 2011;10:372.

10. Moerman F, Lengeler C, Chimumbwa J, Talisuna A, Erhart A, Coosemans M, et al. The contribution of health-care services to a sound and sustainable malaria-control policy. Lancet Infect Dis. 2003;3:99-102.

11. Nankabirwa J, Zurovac D, Njogu JN, Rwakimari JB, Counihan H, Snow RW T, et al. Malaria misdiagnosis in Uganda - implications for policy change. Malar J. 2009;8:66.

12. Kyabayinze DJ, Asiimwe C, Nakanjako D, Nabakooza J, Counihan H, Tibenderana JK. Use of RDTs to improve malaria diagnosis and fever case management at primary health care facilities in Uganda. Malar J. 2010;9:200.

13. Kinney MV, Kerber KJ, Black RE, Cohen B, Nkumah F, Coovadia H, et al. Sub-Saharan Africa's mothers, newborns and children: where and why do they die? PLoS Med. 2010;7:e1000294.

14. Pell C, Menaca A, Were F, Afrah NA, Chatio S, Taylor LM, et al. Factors affecting antenatal care attendance: results from qualitative studies in Ghana, Kenya and Malawi. PLoS One. 2013:8:e53747.

15. Simkhada B, Teijlingen ER, Porter M, Simkhada P. Factors affecting the utilization of antenatal care in developing countries: systematic review of the literature. J Adv Nurs. 2008;619:244-60.

16. Ministry of Health. Health Sector Strategic Plan II, 2005/6-2009/10. Kampala, Uganda: Uganda Printing and Publishing Corporation; 2005.

17. Mbonye A, Birungi J, Yanow S, Magnussen P. Prescription patterns and drug use among pregnant women with febrile illnesses in Uganda: a survey in out-patient clinics. BMC Infect Dis. 2013;13:237.

18. Sangare LR, Weiss NS, Brentlinger PE, Richardson BA, Staedke SG, Kiwuwa MS, et al. Patterns of anti-malarial drug treatment among pregnant women in Uganda. Malar J. 2011;10:152.

19. Odongo CO, Bisaso RK, Byamugisha J, Obua C. Intermittent use of sulfadoxine-pyrimethamine for malaria prevention: a cross-sectional study of knowledge and practices among Ugandan women attending an urban antenatal clinic. Malar J. 2014;13:399.

20. Ribera JM, Hausmann-Muela S, D’Alessandro U, Grietens KP. Malaria in pregnancy: what can the social sciences contribute? PLoS Med. 2007:4:e92.

21. Cochran GW. Sampling techniques. 2nd ed. New York: John Wiley and Sons, Inc; 1963.

22. De Beaudrap P, Turyakira E, White LJ, Nabasumba C, Tumwebaze B, Muehlenbachs A, et al. Impact of malaria during pregnancy on pregnancy outcomes in a Ugandan prospective cohort with intensive malaria screening and prompt treatment. Malar J. 2013;12:139.

23. Lauristen JM. EpiData entry, data management and basic statistical analysis system. Odense, Denmark: EpiData Association; 2006. URL: http://www.epidata.dk.

24. R Core Team. R: a language and environment for statistical computing Vienna, Austria: R Foundation for Statistical Computing; 2013.

25. Anderson TW, Goodman LA. Statistical inference about Markov chains. Annals Math Stat. 1957;28:89-109.

26. Muenz LR, Rubinstein LV. Markov models for covariate dependence of binary sequences. Biomet. 1985;41:91-101.

27. Yeung $S$, White NJ. How do patients use antimalarial drugs? A review of the evidence. Trop Med Int Health. 2005;10:121-38.

28. Ocan M, Bwanga F, Bbosa GS, Bagenda D, Waako P, Ogwal-Okeng J, et al. Patterns and predictors of self-medication in northern Uganda. PLoS One. 2014;9:e92323.

29. Vialle-Valentin CE, Lecates RF, Zhang F, Desta AT, Ross-Degnan D. Predictors of antibiotic use in African communities: evidence from medicines household surveys in five countries. Trop Med Int Health. 2012;17:211-22.

30. Kiwanuka SN, Ekirapa EK, Peterson S, Okui O, Rahman MH, Peters D, et al Access to and utilisation of health services for the poor in Uganda: a systematic review of available evidence. Trans R Soc Trop Med Hyg. 2008;102:1067-74

31. Patouillard E, Hanson KG, Goodman CA. Retail sector distribution chains for malaria treatment in the developing world; a review of the literature. Malar J. 2010;9:50

32. Awor P, Wamani H, Bwire G, Jagoe G, Peterson S. Private sector drug shops in intergreted community case management of malaria, pneumonia and diarhoea in children in Uganda. Am J Trop Med Hyg. 2012;87:92-6. 
33. Chaturvedi HK, Mahanta J, Panday A. Treatment seeking for febrile illness in north-east India: an epidemiological study in the malaria endemic zone. Malar J. 2009;8:301.

34. Ogwal-Okeng JW, Obua C, Waako P, Aupont O, Ross-Degnan D. A comparison of prescription practices between public and private sector physicans in Uganda. East Afr Med J. 2004:S12-6. PMID 15125111.

Submit your next manuscript to BioMed Central and take full advantage of:

- Convenient online submission

- Thorough peer review

- No space constraints or color figure charges

- Immediate publication on acceptance

- Inclusion in PubMed, CAS, Scopus and Google Scholar

- Research which is freely available for redistribution 